\title{
A Statistical Approach for Geometric Smoothing of Discrete Surfaces
}

\author{
Bertrand Kerautret and Achille Braquelaire \\ LaBRI, Laboratoire Bordelais de Recherche en Informatique, \\ UMR 5800, Université Bordeaux 1, \\ 351, cours de la Libération, \\ 33405 Talence, France \\ \{kerautre, achille\}@labri.fr
}

\begin{abstract}
In this article we propose an original method for discrete surface smoothing. This method is based on a statistical estimation of the discrete tangent plane on the voxels of the discrete surface. A geometrical constraint is used to control the recognition of the tangent plane. The resulting surface representation allows us to get both smooth normal vectors of the surface and a smooth surface mesh while preserving the geometrical properties of the surface.
\end{abstract}

Keywords: Digital surfaces, smoothing surface mesh, euclidean nets, discrete normals, visualization, smoothing.

\section{Introduction}

Processing data sets of three-dimensional discrete images brings up the problem of extraction and representation of geometric features, and of visualization of the surface of $3 \mathrm{D}$ objects. The initial volume object can be visualized as a set of 6 connected voxels (also called cuberille representation) [7. But this representation in the discrete space is neither convenient for the analysis of geometric properties of the object nor for the visualization.

A polygonal representation of the boundary of a discrete object is usually used to represent its surface and to perform rendering. One of the first approaches to obtain such a representation was the marching cube algorithm [9]. This method has several drawbacks both from the geometrical and the topological points of view. Other algorithms exist which associate a surface mesh to a discrete surface. For example Türmer and Wütrich triangulate the surfaces by associating centers of voxels to each other [14. Since the direct rendering of the surface obtained after such a triangulation is not smooth, normal vectors are computed in discrete space using a varying neighborhood size [12,13. Then the surface is rendered using Gouraud shading [6]. This rendering technique gives good results, but it smooths only the normal vector of the discrete surface and not the geometry of the surface net. Other methods use deformable models to extract a continuous surface from the original discrete surface [8, 11]. 
An alternative consist in smoothing the object surface by moving the points of the discrete surface. In 2, Braquelaire and Pousset define Euclidean nets as a 3D extension of the model of Euclidean paths [15,3]. In this model, each surface point may be moved inside the unit cube containing it. The smoothing is thus reversible and the original surface can be retrieved from the smoothed one. In the proposed method the points of the discrete surface were moved according to a projection onto some discrete tangent planes. This plane was estimated by searching for local geometric configurations of voxels called tricube [10, 4].

The main drawback of this method is the small neighborhood size which is used to determine the discrete tangent plane. Therefore, the precision of the final result is limited to a local analysis of the discrete surface. In recent works [5], Coeurjolly suggests to use a statistical computation of the discrete tangent plane to obtain the normal vectors of the discrete surface.

In this work we develop this approach and propose a statistical method to recognize accurate tangent plane with a varying neighborhood size. We then use this method to enhance the construction of a smoothed Euclidean net associated with a discrete surface. This method permits to obtain both smooth normal vectors and smooth surface mesh.

In the following section, we introduce the statistical estimation of the discrete tangent plane. In Section 3, we show how to apply a geometric constraint to control the recognition of the discrete tangent plane. Section 4 addresses the problem of transforming the surface from discrete space to a new surface net in continuous space. Afterward, in Section 5 experimental results on both synthetic and real data are presented. Finally, we conclude by future work and implication of this work.

\section{Statistical Estimation of the Tangent Plane's Orientation}

Let us recall some basic definitions in use in this paper. A voxel is a unit cube which the center is an integer point and a surfel is the intersection of two 6adjacent voxels. The surface of a 6 -connected object is the set of surfels adjacent to both a voxel inside and a voxel outside the object. In the same way, a linel is defined as the intersection between two 4-adjacent pixels one of wich belongs to the discrete line. From these definitions surfels and linels are be differentiated according their configurations. Fig. 1 illustrates different types of surfels and linels.

In discrete space, a discrete tangent plane can be defined as the largest discrete plane which can be reconstructed from an initial voxel. The strategy to estimate the discrete tangent plane consists in considering a random draw of surfel. Let us first consider some probalilyties on a discrete line.

Proposition 1. Consider the random draw of linels of type 1 and 2 on a discrete naive line of the first quadrant (i.e. $a>0, b>0$ ). The probability to obtain $a$ linel of type 1 is $\frac{b}{a+b}$, the probability to obtain a linel of type 2 is $\frac{a}{a+b}$. 


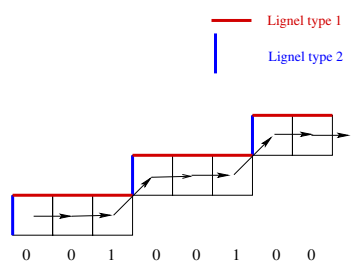

(a)

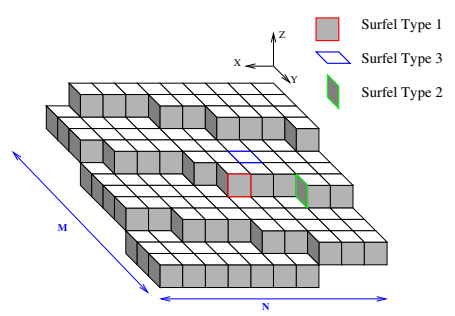

(b)

Fig. 1. Illustration of the different type of linel (a) and of voxels (b)

Proof. Let us consider the discrete naive line represented with the Freeman code. First, we suppose that the discrete line belongs to the first octant (i.e. $a \leq b)$. Then the discrete line is defined by $N$ codes associated to each pixel with directions 0 and 1 (Fig,1 $\mathrm{Fa}$ ). By construction, the probability to obtain the code 1 is $\frac{a}{b}$, then the number of linels of type $1\left(n_{1}\right)$ is $N \frac{a}{b}$. With the same arguments, we can deduce that the number of linels of type $2\left(n_{2}\right)$ is $N$. Thus the two probabilities $P_{1}$ and $P_{2}$ can be written as follows:

$$
\begin{aligned}
& P_{1}=\frac{n_{1}}{n_{1}+n_{2}}=\frac{N}{N\left(\frac{a}{b}+1\right)}=\frac{b}{a+b} \\
& P_{2}=\frac{n_{2}}{n_{1}+n_{2}}=\frac{N\left(\frac{a}{b}\right)}{N\left(\frac{a}{b}+1\right)}=\frac{a}{a+b}
\end{aligned}
$$

The other case for which the discrete line belongs to the second octant (i.e. $b>a$ ) can be inferred by using symmetries.

Now we consider the same process on a discrete plane:

Proposition 2. Consider the process which consists of a random draw of surfels of type 1, 2 and 3 on a discrete naive plane of the first $8^{\text {th }}$ of space (i.e. $a \geq 0$, $b \geq 0, c \geq 0)$. The probability to obtain a surfel of type 1 is $\frac{b}{a+b+c}$, the probability to obtain a surfel of type 2 is $\frac{a}{a+b+c}$ and the probability to obtain a surfel of type 3 is $\frac{c}{a+b+c}$.

Proof. The proof is based on the decomposition of the plane into discrete lines. More precisely, we first suppose that $c=\max (a, b, c)$. We can consider this discrete plane as composition of $N$ discrete lines $\mathcal{D}_{x}(a, c, \mu)$ in the direction of the $\mathrm{x}$ axis and $M$ discrete lines $\mathcal{D}_{y}(b, c, \mu)$ in the direction of the y axis (Fig. 1 $\mathrm{f}$ ). The number of surfels of type $3\left(n_{3}\right)$ is $N M$. Each discrete line $\mathcal{D}_{x}$ generates surfels of type 2 with probability $\frac{a}{c}$. Then the number of surfels of type $2\left(n_{2}\right)$ is $N M \frac{a}{c}$. In the same way, we deduce the number of surfels of type $1\left(n_{1}\right)$ equals to $N M \frac{b}{c}$. Now, we can obtain the following probabilities: 


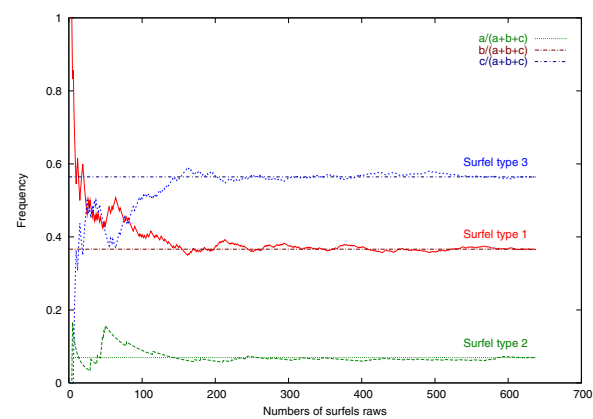

(a)

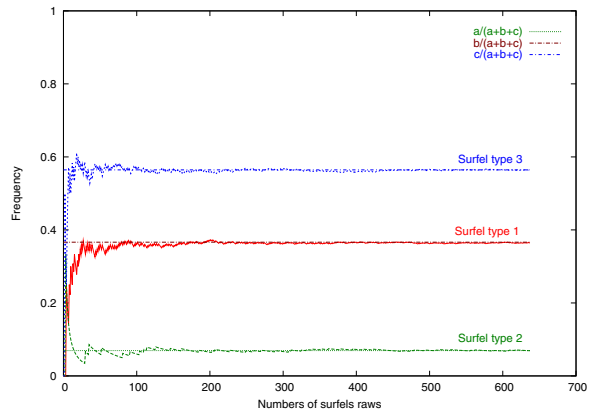

(b)

Fig. 2. Illustration of the convergence of a discrete plane $P(7,17,57,0)$. The graphic (a) was obtained with a random raw of surfels and (b) was obtained with a cubic raw

$$
\begin{aligned}
& P_{1}=\frac{n_{1}}{n_{1}+n_{2}+n_{3}}=\frac{N M\left(\frac{b}{c}\right)}{N M\left(\frac{a+b+c}{c}\right)}=\frac{b}{a+b+c} \\
& P_{2}=\frac{n_{2}}{n_{1}+n_{2}+n_{3}}=\frac{N M\left(\frac{a}{c}\right)}{N M\left(\frac{a+b+c}{c}\right)}=\frac{a}{a+b+c} \\
& P_{3}=\frac{n_{3}}{n_{1}+n_{2}+n_{3}}=\frac{N M}{N M\left(\frac{a+b+c}{c}\right)}=\frac{c}{a+b+c}
\end{aligned}
$$

The other case for which $b=\max (a, b, c)$ and $a=\max (a, b, c)$ can be inferred by using symmetries.

From these probability laws, the coefficients $a, b, c$ can be recovered by solving the system of equation Eq. 3. By considering the number $n_{i}$ of surfel of type $i$, we obtain: $c=k n_{2}, b=k n_{3}, a=k n_{1}$; with $k=\frac{1}{\operatorname{gcd}\left(n_{1}, n_{2}, n_{3}\right)}$.

In order to estimate the parameters of the tangent plane at a point of the discrete surface we have now to traverse the neighborhood of this point in order to analyze the frequency of apparition of the different type surfels. There exists several strategies to select the surfels in the neighborhood of the considered point. We can notice that with a random selection the convergence of the frequencies is quite slow. Fig. 2 shows the evolution of the frequencies with a such draw on a discrete plane of characteristics $\mathcal{P}(7,17,57,0)$. The frequency convergence can be detected after around 450 surfel draws.

A better solution consists in selecting surfels on the boundary of a cubic growing neighborhood from the initial point $P_{0}$. More precisely, the boundary of the cubic neighborhood of size $s$ is defined by:

$$
\begin{aligned}
N_{s}=\left\{P\left(x_{0}+i, y_{0}+j, z_{0}+k\right) \mid\right. & P \in S, \\
& \left.P \text { is 6-connected to } P_{0}, i, j, k \in\{-s, s\}\right\}
\end{aligned}
$$

Fig. 2-b shows the frequency obtained after this selection of surfel. The frequency convergence can be detected with around 100 surfels. This selection of surfels implies a faster convergence of the estimation than the previous method. Other methods of selection were experimented, for example the surfels can be 
selected according to their Euclidean distances from the initial point. Therefore, since this method does not improve the convergence, we have chosen to use the cubic draw of surfels.

Now, we have to define a convergence criterion to detect when the statistical draw follows a probability law. We denote by $f_{i}^{(n)}$ the apparition frequency of a surfel of type $i$ with a neighborhood size equal to $n$. The criterion of recognition $K_{n}$ of the discrete tangent plane can be deduced from this apparition frequencies:

$$
K_{n}=\sum_{i=1}^{6}\left|f_{i}^{(n)}-f_{i}^{(n-1)}\right| \quad \text { with: } f_{i}^{(n)}=\frac{s_{i}^{(n)}}{S_{i}^{(n)}},
$$

where $s_{i}^{(n)}$ represents the number of surfels of type $i$ on the boundary of the cubic neighborhood of size $n$, and $S_{i}^{(n)}$ is the total number of surfels contained in $N_{n}$.

From this criterion, we can define the index $n_{k}$, for which the criterion $K$ reaches a minimum value. Then the parameters of the discrete plane are deduced from the global frequencies $F_{i}^{(n)}$ obtained over the whole neighborhood of size $n_{k}$. More precisely, we have:

$$
F_{i}^{(n)}=\frac{\sum_{j=0}^{n} s_{i}^{(j)}}{S_{\text {Tot }}^{(n)}},
$$

where $S_{T o t}^{(n)}$ represents the total number of all the surfels contained in cubic neighborhood of size $n$.

\section{Position of the Tangent Plane}

When estimating the discrete tangent plane, one needs to determine the parameter $\mu$ associated to the position in space of the discrete tangent plane. This parameter plays an important role to determine the position of the new points in the Euclidean space. All the discrete points $P_{i}$ which belong to the neighborhood of size $n$, need to verify the discrete plane equation:

$$
\mu \leq a x_{i}+b y_{i}+c z_{i}<\mu+\omega
$$

From each point, an interval of possible values of $\mu_{i}$ can be deduced:

$$
\mu_{i} \in\left[\sigma_{i}-\omega, \sigma_{i}[\right.
$$

with $\sigma_{i}=a x_{i}+b y_{i}+c z_{i}$

Since all the discrete points $P_{i}$ do not necessary belong to the discrete plane, we compute the value of $\mu$ for which a maximum of voxels verify the discrete plane equation. If the resulting possible solutions of $\mu$ are defined by an interval $I=\left[\mu_{\min }, \mu_{\max }\right]$, then the solution for $\mu$ is determined as the average value of the two values $\mu_{\min }$ and $\mu_{\max }$.

Now since all the characteristics of the discrete tangent plane are evaluated, it is possible to compute the number of voxels which verify the discrete tangent 
plane equation. The percentage of pixels belonging to the discrete plane is used in association with the previous criterion to limit the neighborhood size extension when ambiguous situation appears. Let us consider for instance a point located at the junction of two planes $P_{1}$ and $P_{2}$ and suppose that each plane has only one kind of surfel. In this case the selected surfels are of two types, one from the plane $P_{1}$ and the other one from $P_{2}$. The statistical analysis does not take into account the local arrangement of surfels and will thus recognize the plane orthogonal to the bisector of $P_{1}$ and $P_{2}$. Such cases may be detected by checking the amount of points which does not belong to the recognized plane. When a tangent plane cannot be recognized the related point may be smoothed by interpolation.

\section{Surface Reconstruction}

From the binary data, a surface mesh is associated to the discrete surface by using the Türmer's algorithm [14]. The resulting surface mesh is obtained by linking together some 6 -adjacent centers of voxel belonging to the discrete surface. The triangulation is determined from different topological configurations of voxel. Fig. 3 $\mathrm{a}$ a illustrates a

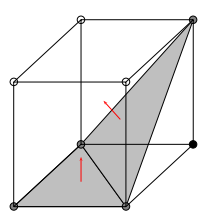

(a)

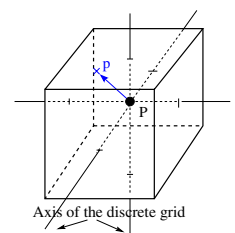

(b)

Fig. 3 sociated triangulation (the voxels belonging to the discrete surface are drawn in light gray). An example of the resulting surface mesh is shown in Fig. 4-a-d. The direct rendering of the surface mesh does not looks as a continuous surface.

To obtain a smooth surface net, all the center of voxels $P$ are projected on the real tangent plane associated to the discrete tangent plane. For this purpose, we consider the upper and lower real tangent planes defined by:

$$
\begin{aligned}
& a x+b y+c z=\mu \\
& a x+b y+c z=\mu+\omega-1
\end{aligned}
$$

From this two planes, we can define the real projection plane as follows:

$$
a x+b y+c z=\mu+\frac{\omega-1}{2}
$$

There are several ways to project the center of a voxel to the associated real tangent plane. A first approach is to project the discrete point according to the three axis. A better solution consists in projecting the center of voxel according the direction of the normal vector. The advantage of such a projection is the possibility to recover the normal orientation from the projected point.

Therefore, if the discrete point $P$ is the origin of the coordinate system, the new point resulting from the projection $p$ is defined by : $p=\alpha(a, b, c)$. The value of $\alpha$ is deduced from the tangent plane equation, we obtain:

$$
\alpha=\frac{\mu+\frac{\omega-1}{2}}{a^{2}+b^{2}+c^{2}}
$$


This projection of discrete point $P$ is illustrated in Fig. 3-b. The new euclidean point $p$ is projected to the tangent plane in the direction of the normal vector. It is possible to show that the projection of the discrete point $P\left(X_{p}, Y_{p}, Z_{p}\right)$ onto the tangent plane satisfies the Euclidean net condition:

$$
\left|x_{p}-X_{p}\right|<\frac{1}{2},\left|y_{p}-Y_{p}\right|<\frac{1}{2} \text { and }\left|z_{p}-Z_{p}\right|<\frac{1}{2}
$$

Each point of the Euclidean net is contained in the unit voxel centered at the associated discrete point. Thus the coordinates of the discrete point can be recovered by rounding the coordinates of the projected point. As a consequence, the transformation of the surface is reversible and the surface mesh is smoothed without any loss in information.

Moreover, from the projected points we can recover the tangent plane orientation by simply computing $a=\sigma\left(x_{p}-X_{p}\right), b=\sigma\left(y_{p}-Y_{p}\right)$ and $c=\sigma\left(z_{p}-Z_{p}\right)$, with $\sigma \in\{-1,1\}$ determined according to the surface orientation of the triangulated surface. Note that a special case appears when the projected point $p$ is merged with the discrete point $P$. To avoid this situation, a solution consists in moving the projected point by an infinite small displacement in order to recover the tangent plane orientation.

Remark that the Euclidean net associated by this method with a discrete surface encode simultaneously the points of the original discrete surface, the points of the smoothed surface and the tangents estimated at each point.

\section{Experiments}

Firstly, we have experimented this method on a synthetic vase. The initial object was obtained after the rasterization of the associated function. Fig. 4 4 a shows the result of the initial polygonal surface obtained directly after the triangulation of the discrete surface. The surface obtained after the projection of the discrete points to the tangent plane is shown in Fig. 4 b. This surface is rendered with flat shading using only the information of the surface mesh. In Fig. 4 c the surface is rendered using the normal vectors recovered from the projection of the Euclidean points and using Gouraud shading. The final surface looks much more smooth than the initial.

To analyze the smoothing effect on the discontinuity of the surface, we have experimented the method on the surface represented in Fig. 44. The object was obtained by sampling a sphere of radius 30 holed by a sphere of radius 15 . As shown in Fig. 4 4 e, the discontinuity between the two spheres is well conserved, while the small sphere is still smooth. Fig. $4 \mathrm{f}$ shows the neighborhood size which is used for the statistical recognition of the discrete tangent plane. The color range from dark blue to white is associated to a size of neighborhood from 1 to 4. This repartition of the neighborhood size shows how the recognition process may detect surface discontinuity.

Then we have analyzed your method on 3D data scan from MRI images. Fig. 5fa shows the initial surface mesh of the discrete data obtained after a 


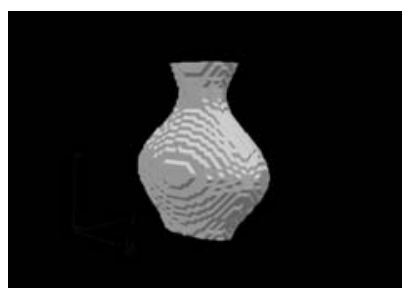

(a)

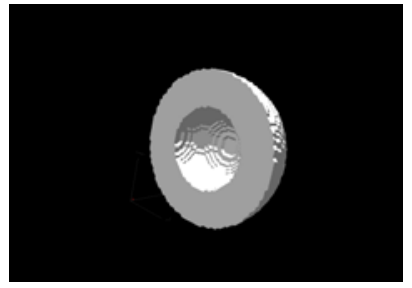

(d)

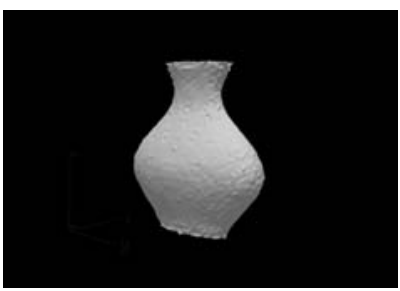

(b)

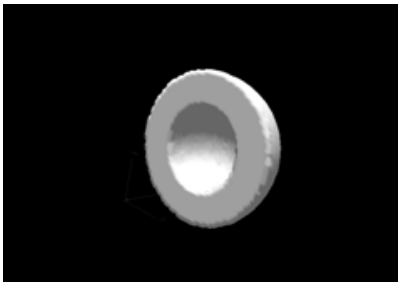

(e)

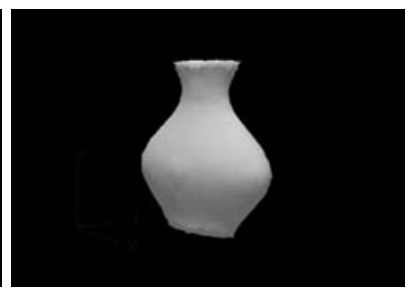

(c)

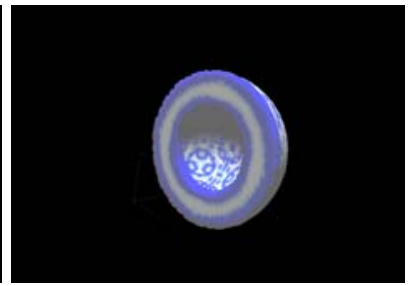

(f)

Fig. 4. Results obtained from synthetic objects. (a) Triangulated surface, (b) smooth surface rendered with flat shading, (c) idem with Gouraud shading, (d) triangulated surface of second object, (e) smoothed surface rendered with Gouraud shading, and (f) neighborhood size used in the statistical recognition

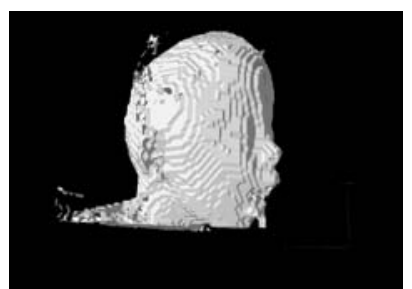

(a)

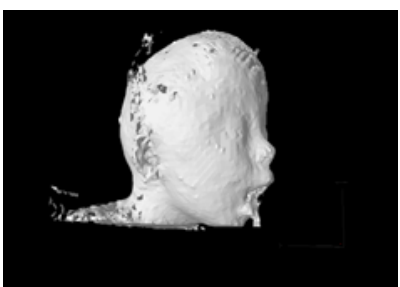

(b)

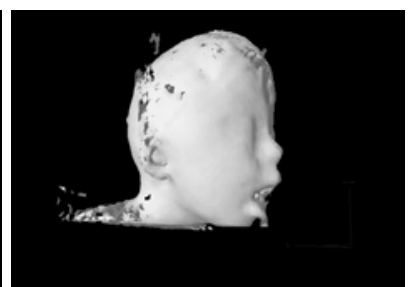

(c)

Fig. 5. Results obtained from the MRI scan of a child head. The binary data was obtained by a simple threshold (on an array of size $58 \times 58 \times 58$ ). Image (a) is the result obtained after the triangulation of the discrete surface. (b) is the resulting surface rendered with flat shading and (c) with Gouraud shading

binary segmentation. The surface was contained in an array of size $58 \times 58 \times 58$. After the application of our method the resulting surface looks very smooth compared to the initial surface and the general shape is well conserved (Fig. 5.b and c). In the same way, Fig. 6 presents results on a more complex topology as the skull. The surface was extracted from a binary array of size $49 \times 49 \times 49$.

Tab. 1 presents quantitative experiments on different discrete spheres. The area of the sphere computed from the Euclidean net was compared to the theoretical value. Another geometrical property was analyzed by the comparison of the distances of each euclidean point to the radius of each sphere. 


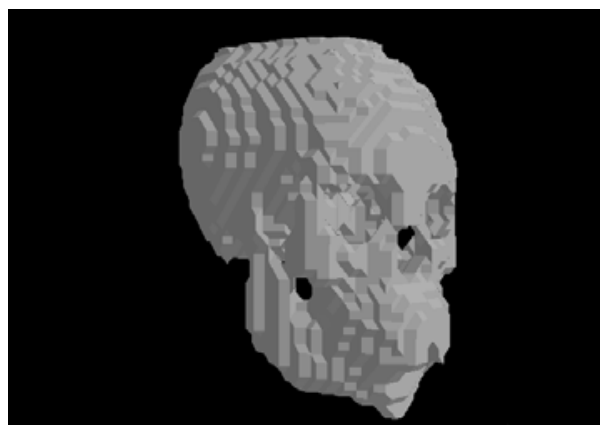

(a)

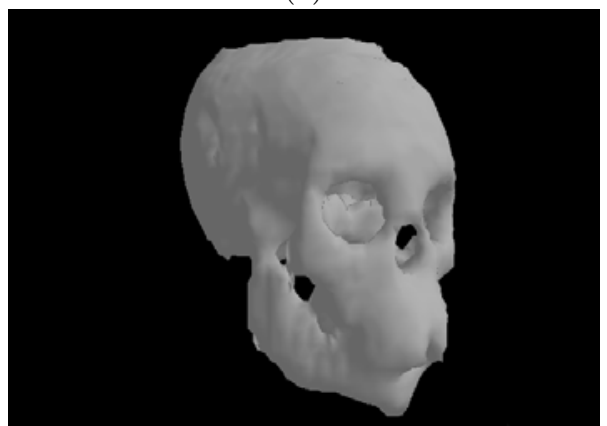

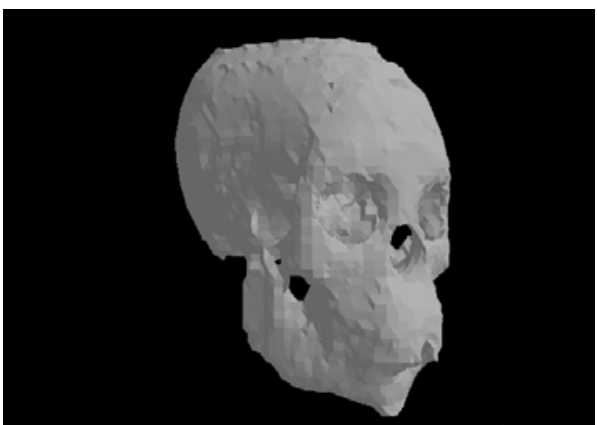

(b)

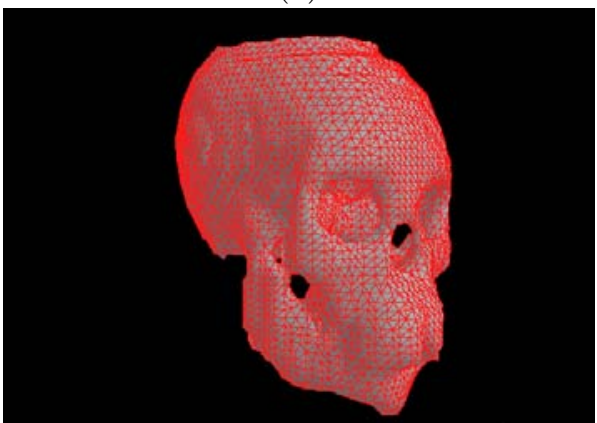

Fig. 6. Results obtained from the MRI scan of a skull. The binary data was obtained by a simple threshold (on an array of size $49 \times 49 \times 49$ ). Image (a) is the result obtained after the triangulation of the discrete surface. (b) is the resulting surface rendered with flat shading and (c) with Gouraud shading

Table 1. Results of geometric properties extraction on discrete sphere with different radius

\begin{tabular}{|c|c|c|}
\hline Radius & area/expected area & mean error distance \\
\hline 10 & 1.020 & 0.097 \\
20 & 1.008 & 0.085 \\
30 & 1.009 & 0.088 \\
40 & 1.007 & 0.087 \\
70 & 1.005 & 0.095 \\
\hline
\end{tabular}

\section{Conclusion and Future Work}

We have introduced a new geometric and statistical method to smooth discrete surfaces. The results of experimentation on both synthetic and real objects show smooth results both on the visual and geometrical points of view. The resulting surface representation can be used for discrete surface rendering and for geometrical properties extraction. 
We are currently working on other methods to define a non symmetric surfel draw. This strategy could be relevant in order to adapt the recognition of the tangent plane when discontinuities on the surface are detected. Moreover, it will be interesting to adapt this method to the inter-voxel boundaries representation. Indeed this representation gives a simple and consistent representation of the discrete surface. Finally, further investigations will concern the use of this smoothing method to improve the reconstruction of the discrete Shape From Shading technique introduced in [1].

\section{References}

1. A. Braquelaire and B. Kerautret. Reconstruction of discrete surfaces from shading images by propagation of geometric features. In Discrete Geometry for Computer Imagery, volume 2886 of LNCS, pages 257-266. Springer-Verlag, 2003.

2. A. Braquelaire and A. Pousset. Automatic and reversible geometric smoothing of the boundary of a discrete 3d object. In Discrete Geometry for Computer Imagery, volume 1953, pages 198-209. Springer-Verlag, 2000.

3. J.P. Braquelaire and A. Vialard. Euclidean paths : a new representation of boundary of discrete regions. Graphical Models and Images Processing, 61:16-43, 1999.

4. J.M. Chassery and J. Vittone. Coexistence of tricubes in digital naive plane. Lecture Notes in Computer Science, 1347:99-110, December 1997.

5. D. Coeurjolly. Algorithmique et géométrie discrète pour la caractérisation des courbes et des surfaces. PhD thesis, Laboratoire ERIC, 2001.

6. H. Gouraud. Continous shading of curved surfaces. IEEE Transaction on Computers, 20(6):623-629, 1971.

7. G.T. Herman and H.K Liu. Three-dimensional display of human organs from computed tomograms. Computer Graphics and Image Processing, 9(1):1-21, 1979.

8. J.-O. Lachaud and A. Montanvert. Deformable meshes with automated topology changes for coarses-to-fine 3d surface extraction. Medical Image Analysis, 3(2):187$207,1999$.

9. W.E. Lorenson and H.E. Cline. Marching cubes: A height resolution 3d surface reconstruction algorithm. volume 21, pages 11-118, 1987.

10. J.M. Schramm. Coplanar tricubes. Lecture Notes in Computer Science, 1347:8798, December 1997.

11. D. Terzopoulos, A. Witkin, and M. Kass. Constraints on deformable models: Recovering 3d shape and nonrigid motion. Artificial Intelligence, 36:91-123, 1988.

12. G. Thürmer. Smoothing normal on discrete surfaces while preserving slope discontinuities. Computer Graphics Forum, 20(2):103-114, 2001.

13. G. Thürmer and C. A. Wütrich. Varying neighbourhood parameters for computation of normals on surfaces in discrete space. In IEEE Computer Society Press, editor, Computer Graphics International, pages 616-625, 1998.

14. G. Thürmer and C.A. Wütrich. Polygon mesh generation for discrete surfaces in 3d space. In Eighth Eurographics Workshop on Visualisation in Scientific Computing, pages 117-126, 1997.

15. A. Vialard. Chemins euclidiens : Un modèle de représentation des contours discrets. Phd thesis, Université Bordeaux 1, 1996. 